\title{
Hybrid PET/MR Imaging of the Heart: Potential, Initial Experiences, and Future Prospects
}

\author{
Christoph Rischpler, Stephan G. Nekolla, Isabel Dregely, and Markus Schwaiger \\ Nuklearmedizinische Klinik und Poliklinik, Technische Universität München, Munich, Germany
}

\begin{abstract}
Learning Objectives: On successful completion of this activity, participants should be able to describe (1) advantages and shortcomings of hybrid PET/MR scanners with respect to cardiovascular applications (e.g. myocardial perfusion imaging and viability imaging), (2) additional value of the MR component in cardiac imaging, and (3) technical challenges and workflow considerations regarding hybrid PET/MR scanners in the field of cardiology (e.g. attenuation correction and cardiac/respiratory/patient motion).
\end{abstract}

Financial Disclosure: Dr. Schwaiger is an investigator, meeting participant, and lecturer for Siemens Medical. The authors of this article have indicated no other relevant relationships that could be perceived as a real or apparent conflict of interest.

CME Credit: SNMMI is accredited by the Accreditation Council for Continuing Medical Education (ACCME) to sponsor continuing education for physicians. SNMMI designates each JNM continuing education article for a maximum of 2.0 AMA PRA Category 1 Credits. Physicians should claim only credit commensurate with the extent of their participation in the activity. For CE credit, participants can access this activity through the SNMMI Web site (http:// www.snmmi.org/ce_online) through March 2016.

PET/CT and other combined scanners have in the past decade rapidly emerged as important research tools and are proving to be invaluable for improved diagnostics in routine nuclear medicine. The design of hybrid PET/MR scanners presented a formidable technical challenge, and only recently were these instruments introduced to the market. Initial expectations of the performance of these scanners have been high, notably because of the potential for superior tissue contrast inherent in the MR modality, as well as the potential for multiparametric functional imaging in conjunction with PET. However, the additional value and potential clinical role that these new systems might bring to the cardiac field have yet to be documented. This review presents a comparative summary of the existing applications for PET and MR in the field of cardiology and suggests potential cardiac applications exploiting unique properties of the newly introduced combined instrumentation.

Key Words: PET; MR; hybrid imaging; cardiology

J Nucl Med 2013; 54:402-415

DOI: 10.2967/jnumed.112.105353

$\mathbf{T}$ he diverse range of imaging modalities for cardiology includes echocardiography, CT, MR imaging, SPECT, and PET, each of which offers distinct properties and advantages. The demand for combined PET/CT instrumentation in clinical nuclear medicine has grown remarkably, because of the notable advantages presented by hybrid imaging with respect

Received Nov. 26, 2012; revision accepted Feb. 1, 2013.

For correspondence or reprints contact: Markus Schwaiger, Department of Nuclear Medicine, Technische Universität München, Klinikum rechts der Isar, Ismaninger Strasse 22, 81675 Munich, Germany.

E-mail: markus.schwaiger@tum.de

Published online Feb. 12, 2013.

COPYRIGHT (C 2013 by the Society of Nuclear Medicine and Molecular Imaging, Inc. to anatomic localization of lesions. Although these advantages were initially driven by the demands of oncology imaging, the resultant broader availability of PET/CT has provided the opportunity to use these scanners for indications beyond oncology, such as in the field of cardiac imaging (1). Furthermore, CT images are necessary for rapid attenuation correction, which is of particular importance for quantification in myocardial perfusion imaging. Through the use of suitable CT components and modification of imaging protocols, we can now visualize coronary anatomy by CT angiography, in conjunction with functional and metabolic imaging of the heart by PET, which together provide enhanced diagnostic information for the clinician (2). Drawing on this experience with PET/CT, we present in this review technical aspects of the design and performance of the newly available PET/MR scanners and summarize the existing comparative studies of PET and MR imaging in cardiology, as the basis for predicting the impact of hybrid PET/MR scanners in diagnostics and therapeutics for cardiology.

\section{STRENGTHS AND WEAKNESSES OF MR VERSUS CT AS AN ADJUNCT TO PET}

The major advantages of MR over CT are the higher softtissue contrast and the lack of radiation exposure to the patient. Morphologic information; attenuation correction maps-essential for regional quantification of PET-based physiologic parameters; and all other information is acquired without ionizing radiation by MR. Different approaches to reduce the radiation dose from $\mathrm{CT}$ were undertaken; nonetheless, CT-based attenuation correction maps still add, for example, about $0.8 \mathrm{mSv}$ to a cardiac stress-rest scan (3).

At the same time, the electromagnetic fields necessary for MR imaging represent a major disadvantage, especially in cardiac imaging. A substantial number of patients cannot be imaged using MR because of the presence of pacemakers, implantable cardioverter defibrillators, or mechanical 
heart valves. In these patients, PET/CT proves to be an invaluable alternative for noninvasive cardiac imaging.

Despite the disadvantage of ionizing radiation, however, CT has become the most commonly used technique for noninvasive coronary angiography. It is fast and easy to perform. Also, the use of high-pitched prospectively electrocardiogram-triggered coronary CT angiography with low voltage has reduced the radiation dose to about 1.3 $\mathrm{mSv}$ (4). Although there has been considerable progress in MR angiography since the initial published reports, MR acquisition remains for the present a technical challenge due to artifacts arising from cardiac, respiratory, and patient motion and the need for high spatial resolution while retaining high-vessel-to-tissue contrast, and high signal-to-noiseratio, all within a reasonable scanning time.

For the assessment of left ventricular (LV) function, MR imaging represents the gold standard and has been extensively validated. MR shows great accuracy in the determination of functional parameters such as LV volumes, mass, ejection fraction, regional wall motion, and myocardial thickening (5). Although CT-based assessment of LV function correlates well with MR, radiation exposure to the patient still represents a major issue (6).

Another important point that needs to be addressed is the application of contrast medium. Most patients tolerate MR contrast agents well, and acute adverse reactions seldom occur. Furthermore, a rare condition called nephrogenic systemic fibrosis occurs almost exclusively in patients with acute renal failure or severe chronic kidney disease. Iodinated CT contrast agents may lead to a condition called contrast-induced nephropathy, which is a leading cause of iatrogenic acute kidney failure. Furthermore, severe adverse reactions occur more frequently after the application of CT contrast medium. Notably, MR perfusion imaging can also be accomplished without the use of contrast agents, by determining the $\mathrm{T} 1$ for both myocardium and blood, a technique called arterial spin-labeled MR imaging (7). Even though this technique proved to have the potential to assess myocardial perfusion without any contrast medium, an advantage that might be especially valuable in patients with end-stage renal disease or when serial measurements are needed, it is far from routine clinical practice (8). Although initial studies for CT myocardial perfusion showed promising results, 64-slice multidetector CT isaccording to a recent study_not feasible for detecting ischemia (9).

Although molecular information is hard to assess by CT, as images are based simply on differences in x-ray attenuation, MR offers promising features of molecular imaging, such as spectroscopy. Spectroscopy allows the noninvasive investigation of myocardial metabolism and composition. Using ${ }^{1} \mathrm{H}-\mathrm{MR}$ spectroscopy for the regional quantification of creatine content, viable myocardium can be distinguished from nonviable (scarred) tissue (10). This technique also allows measurement of the triglyceride content of myocardium, and interestingly, high myocardial lipid content was linked to low septal wall thickness and high LV mass (11). Furthermore, ${ }^{23} \mathrm{Na}$ MR spectroscopy is a promising application for myocardial viability assessment and the detection of myocardial ischemia (12). Another interesting feature of MR is diffusion spectrum MR imaging tractography. This imaging modality was first implemented for brain research but also yields great potential as a research tool for cardiology. This method allows imaging of the architecture of the organization of myocardial fibers with exceptional resolution (13). Using this technique, Sosnovik et al. revealed the presence of meshlike networks of orthogonal myofibers within the infarcted rat myocardium (14).

Finally, CT is fast and easy to perform, whereas MR protocols are often time-demanding and require good patient compliance. Furthermore, especially for the newly developed imaging protocols for PET/MR, excellent interaction of well-trained personnel from different subspecialties is a prerequisite. Table 1 gives an overview of general strengths and weaknesses of MR versus CT as an adjunct to PET.

\section{TECHNICAL REQUIREMENTS FOR PET/MR}

\section{Attenuation Correction}

The introduction of hybrid PET/MR tomographs into the medical imaging field is so recent that it is difficult to judge the merits of this technology in comparison with PET/CT or standalone MR imaging (15,16). At present, 2 different architectures are commercially available: the Ingenuity TF PET/MR (Philips) combines currently available PET and MR imaging devices along a common rail system (17), whereas fully integrated systems, such as the Biograph mMR (Siemens), entail a compact PET system installed within the 70-cm bore of a conventional 3-T MR device (18). Both designs lack the previously used methods for attenuation correction, that is, a rotating radionuclide source as in stand-alone PET systems or a dedicated CT component as in hybrid PET/CT systems. Thus, the attenuation map for 511-keV photons must be generated by other means, as there is no direct, physical relation between MR images and the attenuation coefficients of the imaged

TABLE 1

General Strengths and Weaknesses of MR Versus CT as Adjunct to PET

\begin{tabular}{lcc}
\hline \multicolumn{1}{c}{ Parameter } & CT & MR \\
\hline Radiation & - & Not applicable \\
\hline Electromagnetic field & Not applicable & - \\
Contrast agents for CT & - & - \\
Handling & + & - \\
Soft-tissue contrast & - & +++ \\
Bone imaging & +++ & - \\
\hline LV function & - & +++ \\
Noninvasive angiography & +++ & + \\
Molecular imaging potential & - & ++ \\
\hline
\end{tabular}


tissue. However, PET quantification is impossible without a $\mu$-map. In particular, a map containing the attenuation coefficients for $511-\mathrm{keV}$ photons at each voxel within the entire imaged volume is required. The calculation of this attenuation map presents a major challenge for PET/MR (19) in general, and imperfections in the $\mu$-map can substantially degrade cardiac PET results, as is known from cardiac PET/CT (20).

Several MR approaches for attenuation correction are currently used, based on segmentation, templates or atlases, or the PET emission data (Table 2). For segmentation-based approaches, the attenuation map is segmented into different tissue classes with a fixed attenuation coefficient per tissue class, as first proposed by Huang et al. in 1981 (21). Building on this well-established concept, our group proposed to use segmented MR-based attenuation correction for wholebody PET/MR imaging (22). Using water-weighted and fatweighted images computed from a Dixon MR sequence (23), we classify each voxel as air, lungs, fat, or soft tissue. This scan requires typically $18 \mathrm{~s}$ per bed position, measured during a single breath-hold. Cortical bone is almost impossible to segment from these data and thus, in analogy to segmented whole-body data from PET-only devices, is ignored. In an alternate approach, T1-weighted turbo spinecho sequences are used for attenuation correction. Bone is also ignored by this approach, but this sequence allows a shorter imaging time at the expense of not differentiating between fat $\left(\mu=0.086 \mathrm{~cm}^{-1}\right)$ and soft tissue $(\mu=0.096$ $\mathrm{cm}^{-1}$ ) (24). The limitations of segmentation-based techniques arise from the fixed number of tissue classes that can be discriminated and, in the context of heart imaging, from the use of a fixed attenuation coefficient for lung tissue, which is known to vary between patients and locations in the lung. In addition, ignoring bone obviously results in an underestimation of tissue attenuation, especially near the skeleton. Tumor uptake in PET/MR has differed from that in PET/CT in the range of $5 \%-15 \%(22,24)$ or up to $23 \%$ (25). Furthermore, metal implants result in a void of MR signal and do not contribute to the attenuation map. In addition, the effect of MR contrast agents can be problematic because of effects on segmentation of fat, lung, and soft tissue arising from reduced T1 values (26). Despite these considerations, the segmentation approach is rather stable and computationally fast and has been implemented in commercially available PET/MR systems. The Biograph mMR uses a modification of this approach (22), and our group has shown a good correlation between PET/CT and PET/MR in oncologic studies (27). The sequential Ingenuity TF PET/MR likewise uses a version of this approach (24).

Template-based approaches match a model to the patient's anatomy such that the known attenuation map of the model is modified to match the target. In the particular case of head imaging, a rigid model will suffice, whereas for whole-body applications, the need for an elastic registration presents an obstacle to implementation for routine use. In addition, atlas-based methods might fail if anatomic abnormalities are present. A recent implementation of a combined template and segmentation approach has been shown to be feasible in whole-body imaging (28).

In emission-based approaches to attenuation correction, the recorded PET data themselves might provide the information needed to calculate an attenuation map. However, the effect of photon scatter limits the applicability of this approach (29). Still, it bears consideration that information derived from emission data was well suited for motion correction in cardiac imaging (20), and Nuyts et al. suggested

TABLE 2

Four Approaches to Attenuation Correction, with Practical Goal of Combining All

\begin{tabular}{|c|c|c|c|c|}
\hline Technique & Method & $\begin{array}{c}\text { Implemented on } \\
\text { commercial systems }\end{array}$ & Advantages & Limitations \\
\hline $\begin{array}{l}\text { MR image } \\
\text { segmentation }\end{array}$ & $\begin{array}{l}\text { MR image segmentation } \\
\text { into tissue classes and } \\
\text { default attenuation } \\
\text { coefficients }\end{array}$ & Yes & $\begin{array}{l}\text { Relatively simple } \\
\text { to implement } \\
\text { and fast execution }\end{array}$ & $\begin{array}{l}\text { MR imaging field } \\
\text { of view is smaller } \\
\text { than PET field of } \\
\text { view; thus, truncation } \\
\text { might occur }\end{array}$ \\
\hline $\begin{array}{l}\text { Atlas or } \\
\text { template-based }\end{array}$ & $\begin{array}{l}\text { Estimation of tissue using } \\
\text { atlas of reference } \\
\text { patient morphology }\end{array}$ & No & $\begin{array}{l}\text { Useful in situations in } \\
\text { which MR images } \\
\text { are distorted or } \\
\text { lacking }\end{array}$ & $\begin{array}{l}\text { Nonlinear registration in } \\
\text { whole-body imaging } \\
\text { between patient and } \\
\text { model is nontrivial }\end{array}$ \\
\hline PET emission-based & $\begin{array}{l}\text { Tissue estimation } \\
\text { based on emitted } \\
\text { annihilation photons }\end{array}$ & Yes & $\begin{array}{l}\text { Useful in situations in } \\
\text { which MR images } \\
\text { are truncated }\end{array}$ & $\begin{array}{l}\text { Method requires significant, } \\
\text { unspecific background } \\
\text { tracer uptake, which } \\
\text { might be small with } \\
\text { targeted agents }\end{array}$ \\
\hline $\begin{array}{l}\text { Integration of } \\
\text { hardware }\end{array}$ & $\begin{array}{l}\text { Addition of previously } \\
\text { measured (e.g., } \\
\text { with CT) hardware } \\
\text { attenuation maps }\end{array}$ & Yes & $\begin{array}{l}\text { Independent of MR } \\
\text { imaging if location } \\
\text { is known }\end{array}$ & $\begin{array}{l}\text { Method requires precise } \\
\text { information on location }\end{array}$ \\
\hline
\end{tabular}


using this approach to recover truncated parts of an attenuation map (30).

All techniques present their own advantages and disadvantages, and one might suppose that a future optimization of attenuation correction for PET/MR may entail a combination approach. Such a combination of segmentation- and emission-based approaches has already been implemented in the Biograph mMR, for which the field of view of the MR imaging system is only $45 \mathrm{~cm}$. This may result in truncation of the attenuation map, as the patient's arms are typically aligned along the body axis during scanning $(30,31)$. However, no extended validation is yet available for this approach. The most obvious solution to this problem would be to extend the field of view, as was done in a recent study with promising results (32). An example of attenuation correction maps generated by $\mathrm{CT}$ and $\mathrm{MR}$ and the resulting attenuation-corrected PET images of a patient who was referred to our institution for a viability scan is depicted in Figure 1.

Finally, an additional source of scatter and attenuation needs to be included: the variety of MR imaging-related components located within the field of view of the PET detectors. These objects include the patient bed, which is significantly more massive than beds typically used in PET/ $\mathrm{CT}$, as well as the various fixed coils and variable-surface coils. Other interfering objects include positioning aids, patient monitoring devices, cables, and earphones, all of which are sources of photon scatter and attenuation. The fact that these components are invisible in conventional MR sequences and thus will not contribute to the MR-based attenuation maps potentially affects image homogeneity and quantification.

The contribution of the various components differs in magnitude, and typically only the bed and those fixed coils for which the spatial location is accurately known are included in precalculated attenuation models (33). Hardware vendors redesigned the hardware components in order to minimize their impact, but their effects are still measurable $(26,34)$, albeit of uncertain impact on diagnostic scans in larger patient cohorts. In the specific case of cardiac imaging, the preliminary data now available point to a good general agreement between PET/CT and PET/MR in

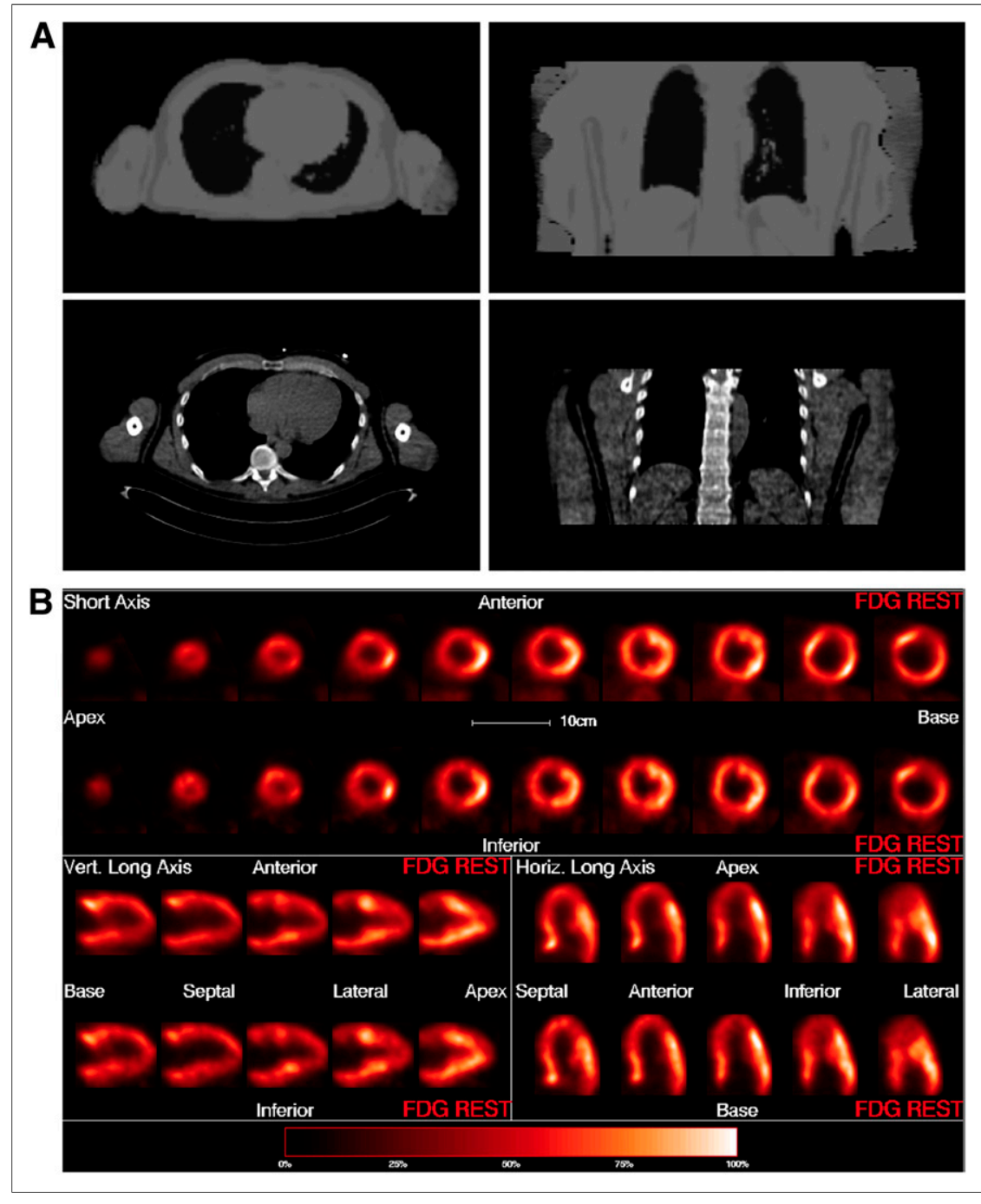

FIGURE 1. Attenuation correction maps (A) generated by MR (top) and CT (bottom), as well as resulting attenuation-corrected ${ }^{18}$ F-FDG PET images (B) based on MR attenuation correction map (top row of short- and long-axis images) and on CT attenuation correction map (bottom row of short- and long-axis images). In MR-based attenuation correction maps, truncated parts of arms were successfully recovered but $\mu$-values are somewhat inhomogeneous. Patient was referred to our institution for ${ }^{18} \mathrm{~F}-\mathrm{FDG}$ viability testing. 


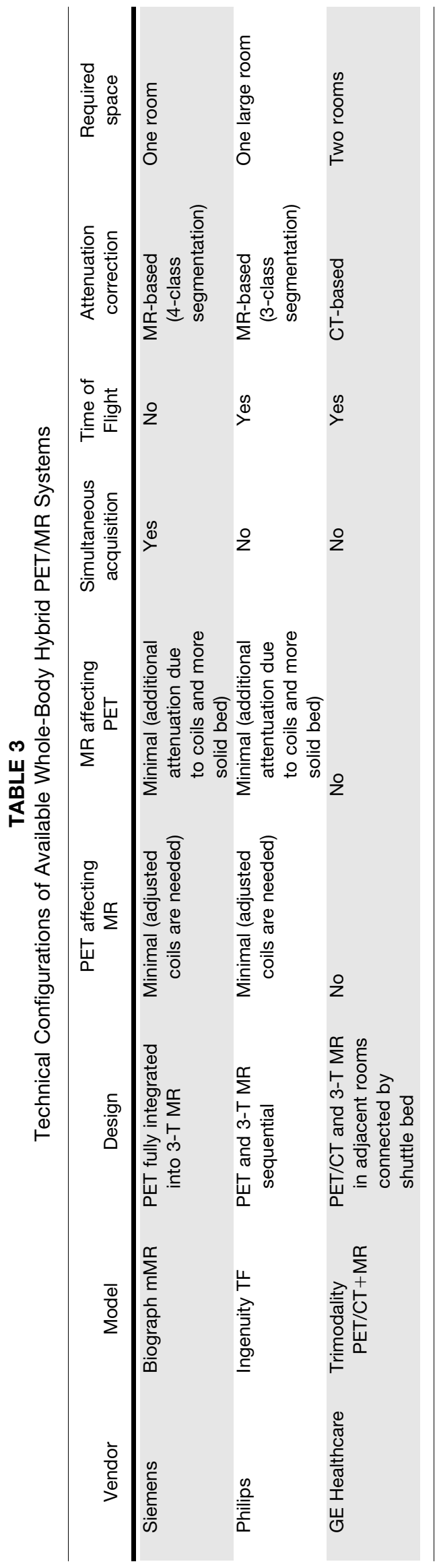

the left ventricle, but differences in regional quantification that were found (35) affect where the attenuation correction map is truncated in particular patients (Table 3 shows the technical configurations of available PET/MR systems).

\section{Workflow Considerations}

Attenuation correction is a necessary prerequisite for PET image quantification, and consequently, the alignment between emission and "transmission," however obtained, is sine qua non. As established especially for cardiac PET/CT, correct alignment is crucial to avoid false-positive findings arising from absolute motion of the patient between the 2 scans, as well from the intrinsic motion of the heart and thorax due to shifting of the body (20). As a matter of convenience, MR attenuation scans can easily be repeated in cases of substantial motion, as no ionizing radiation is involved. Furthermore, on the basis of our experience in several dozen examinations to date, the alignment between the MR attenuation correction, acquired in $18 \mathrm{~s}$ during expiration, and the PET, acquired over many respiratory cycles, matches well in almost all cases.

Although these considerations are relevant for serial PET/MR imaging acquisitions, the same motions occur during fully simultaneous acquisitions. It should be recalled that MR imaging is, to date, usually a serial imaging technology, in which the individual images encompassing a data volume are acquired sequentially, with acquisition times per slice ranging from $50 \mathrm{~ms}$ for perfusion scans to several breath-holds for high-resolution MR images. In contrast, PET acquisitions are fully volumetric, with typical frame lengths ranging from $5 \mathrm{~s}$ to $20 \mathrm{~min}$, depending on the protocol; frame duration depends ultimately on the radiotracer concentration-that is, counting rates. Another consideration in cardiac hybrid PET/MR imaging is that highresolution scans are acquired over many breath-holds. Because the reproducibility of breath holding is limited, the construction of a data volume from slices of shifting position is challenging. However, because the strategy of serial breath-holds is also rather inconvenient for the patient, the MR community is actively developing so-called freebreathing sequences, wherein motion sensors trigger the scan by detecting actual patient motion (36) or motion correction software is applied (37). This new kind of sequence is expected to improve significantly the alignment between PET and MR imaging data, thus facilitating synergistic reading. Simultaneous imaging protocols might be applied in myocardial perfusion imaging. The combination with PET tracers of well-described physiologic properties, good volume coverage, and only moderate spatial resolution could be supplemented with high-resolution first-pass MR imaging, with only limited spatial coverage and suboptimal contrast medium properties. In this case, both tracer and contrast medium are extracted under the same cardiac stress conditions in a time window of typically $60 \mathrm{~s}$, although the injection times will differ (MR imaging: $5 \mathrm{~s}$; PET: $30 \mathrm{~s}$ ), and the injections must be carefully synchronized. The temporal domains differ to 
a greater extent, and the gating logic is inherently different (prospective vs. retrospective). Whereas high-resolution scans such as cine MR imaging and late gadolinium enhancement (LGE) are acquired in less than $5 \mathrm{~min}$, PET recordings used for an electrocardiogram-gated reconstruction will take up to $20 \mathrm{~min}$. Thus, in patients with greater heart rate variability, the contractile information potentially differs between modalities. A technical solution synchronizing the contraction for an optimized display is therefore desirable.

In summary, truly simultaneous PET/MR protocols present formidable technical difficulties, and perfectly matching frames are not yet automatically achievable. Consequently, misalignment arising from patient motion is an issue requiring close attention. However, in hybrid PET/MR scanners, real-time MR-based motion correction and partial-volume correction may eventually become available.

From the perspective of workflow, parallel PET/MR imaging, here in contrast to the term simultaneous, presents a significant advantage over sequential acquisition of PET and MR imaging. The latter is inconvenient for the patient and logistically demanding and is also time-consuming, as both examinations typically require 30-60 min, depending on the PET tracer and complexity of the MR protocol. Consequently, parallel PET/MR acquisitions could improve compliance in patients, especially those whose underlying disease does not permit breath holding, and furthermore, higher throughput would improve cost-effectiveness. A potential workflow is depicted in Figure 2.

Another important aspect of economic considerations is that advances in CT scanners have historically been driven by hardware changes. Thus, new generations of CT technology regularly generate the need for expensive acquisitions of PET/CT scanners. In contrast, advances in MR imaging are often accomplished by the generation of new sequences and external coils, making the upgrading of existing hardware more affordable. However, hybrid imaging devices increase the demands on personnel, both for operating the scanner and for interpreting the images. Here, a close collaboration of nuclear medicine physicians, radiologists, and cardiologists is crucial. This holds especially true for the MR imaging component, because it is more interactive and has greater complexity than does CT for disease- or patient-specific adjustments in the imaging sequences, as pertains to such factors as the availability of different image contrast weightings, spatial coverages, sources for potential artifacts (image geometry, flow, chemical shift), and timings to optimize delayed-enhancement imaging.

\section{INITIAL EXPERIENCE AND POTENTIAL APPLICATIONS Coronary Artery Disease (CAD) Detection and Characterization}

In cardiology, PET imaging is still most frequently used for the diagnosis of obstructive CAD. This method yields high sensitivity and specificity of about 90\% (38-40) and is of proven value for accurate prognosis and good patient management (41-43). In particular, the extent of ischemia as determined by PET myocardial perfusion imaging has prognostic implications when patients are assigned to the optimal therapy, either revascularization or intensified medical therapy (44). The MR modality has great promise in the diagnosis of obstructive CAD too; coronary artery stenosis can be detected by first-pass MR imaging after injection of a fast bolus of gadolinium contrast agent such as gadolinium-diethylenetriaminepentaacetic acid (DTPA) and yields sensitivity and specificity of about $91 \%$ and $81 \%$, respectively (45-47).

PET myocardial perfusion imaging presents the advantage of allowing myocardial blood flow, and secondarily coronary flow reserve, to be quantified by tracer kinetic modeling. Absolute myocardial blood flow quantification is of special importance in patients with extensive CAD, in whom reduced perfusion may be missed or underestimated by simple visual or semiquantitative analysis in SPECT myocardial perfusion imaging, because of the phenomenon of balanced ischemia $(48,49)$. On the other hand, subclinical changes in the vasoreactivity of the coronary arteries, as well as treatment response or progression, may be identified using this PET feature (50). Among

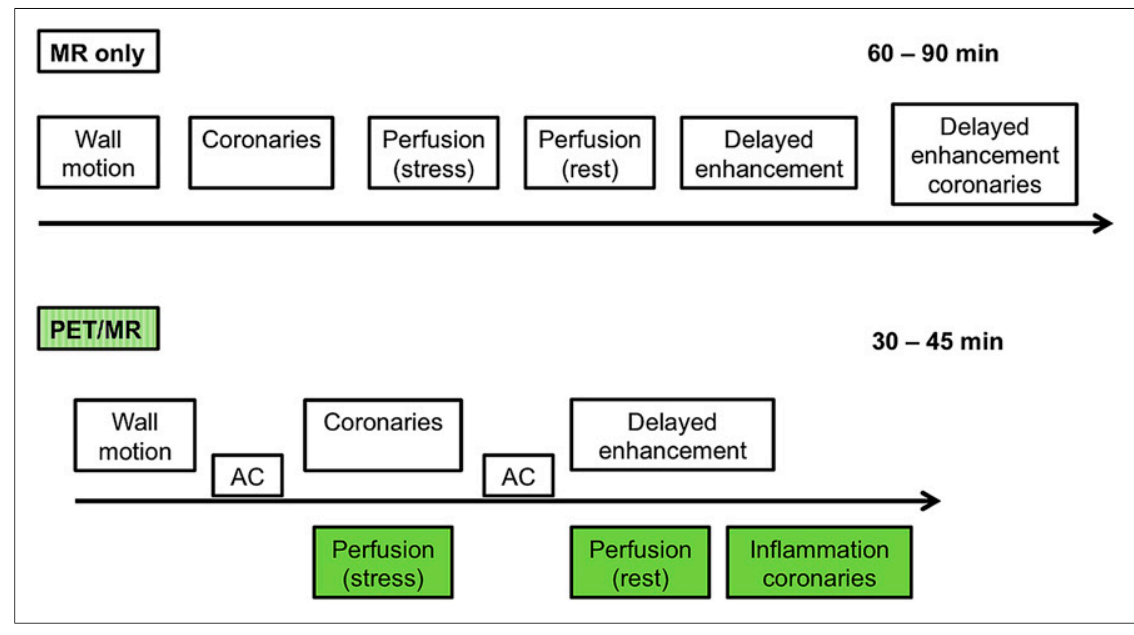

FIGURE 2. Possible workflow for comprehensive PET/MR cardiac study in which demand in instrument time and labor might be reduced and work efficiency increased. MR imaging studies are shown in white boxes; PET studies are shown in green boxes. 
the available PET tracers for myocardial perfusion are ${ }^{13} \mathrm{~N}$-ammonia $\left({ }^{13} \mathrm{NH}_{3}\right),{ }^{15} \mathrm{O}$-water, ${ }^{82} \mathrm{Rb}$, and ${ }^{18} \mathrm{~F}$-flurpiridaz, with the last of these currently being evaluated in clinical phase 3 trials (51).

Although the quantification of myocardial perfusion by PET is well validated and is in routine use at many centers, the usefulness of cardiac MR imaging for flow quantification is still under investigation. In one of the few published reports, Schwitter et al. demonstrated that MR imaging can quantify myocardial perfusion deficits in patients with CAD (52). A flow index using a pixelwise upslope parameter of the signal intensity curve during gadolinium-DTPA transit was calculated. Using this index, sectors defined as pathologic by MR showed a linear correlation with ${ }^{13} \mathrm{NH}_{3}$ PET-defined pathologic sectors (slope $=0.94, r=0.76$, $P<0.0001$ ). Subsequently, Ibrahim et al. determined for MR imaging a pathologic stress-rest index threshold, which was low compared with validated PET thresholds (1.3 vs. 2.5) (53).

However, both modalities hold several shortcomings: the underestimation of the MR-based stress-rest index when compared with the ${ }^{13} \mathrm{NH}_{3}$ PET results is one of several hurdles to be overcome before cardiac MR can find routine use. Other relevant issues of cardiac MR include the possibility of image-derived assessment of the arterial input function, the rather low distribution volume of gadolinium-DTPA, the rapid diffusion of gadolinium-DTPA into the extracellular space (which poses difficulties for kinetic modeling), and the limited spatial coverage of the left ventricle, which is imaged in 3-5 slices. One of the shortcomings of PET is that no morphologic information but only information on perfusion abnormalities is gained. Consequently, it is not possible to differentiate between a reduced perfusion that is due to epicardial coronary stenosis and one that is due to microvascular dysfunction. Furthermore, a reduced perfusion - being relative or quantitative - is not always indicative of myocardial scarring, especially in patients with dilated cardiomyopathy (54).
Simultaneous PET/MR acquisition now allows the direct comparison of the diagnostic performance of each modality at identical resting and stressed conditions and thus can help with the cross-validation and refinement of the applied techniques and the diagnostic performance of both techniques. Furthermore, morphologic information gained by MR, such as MR angiography or LGE, together with functional imaging gained by PET, such as perfusion, would potentially help with differentiating between epicardial stenosis and microvascular dysfunction or between a scar and dysfunctional but viable myocardium. Figure 3 presents a case from our institution, in which myocardial perfusion was simultaneously assessed with PET and MR using the Biograph $\mathrm{mMR}$, a fully integrated system.

\section{Evaluation of Known or Suspected Cardiomyopathy \\ Differentiation of Ischemic from Nonischemic Cardiomy-} opathy. Classification of patients with heart failure is based on the etiology of their disease. For state-of-the-art treatment and accurate prognosis, noninvasive, imagebased differentiation of cardiomyopathies is important. Although no single imaging modality provides all the necessary information, PET and MR each offers distinct benefits for patient workup.

Different approaches using PET imaging have been described. Eisenberg et al. were able to differentiate between both disease entities with high sensitivity and specificity by the assessment of myocardial fatty acid consumption using ${ }^{11} \mathrm{C}$-palmitate (55). The technique most often applied, however, is the combined assessment of myocardial perfusion (e.g., ${ }^{13} \mathrm{~N}$-ammonia, ${ }^{82} \mathrm{Rb}$ ) and glucose metabolism ( ${ }^{18}$ F-FDG) by PET. Because of its high accuracy and reproducibility, MR is the gold standard for the assessment of cardiac structure, LV function, and wall motion. Furthermore, MR allows scar delineation (LGE imaging), identification of fat (T1-weighted black blood with fat suppression), tissue edema and inflammation (T2-weighted sequences), or iron content of the tissue

FIGURE 3. Simultaneous acquisition of myocardial perfusion using Biograph mMR, a fully integrated PET/MR system. This dynamic illustration shows ${ }^{13} \mathrm{NH}_{3}$ PET perfusion (top), gadolinium-DTPA MR perfusion (bottom), and coregistration of both modalities (middle) at mid-ventricular slice.

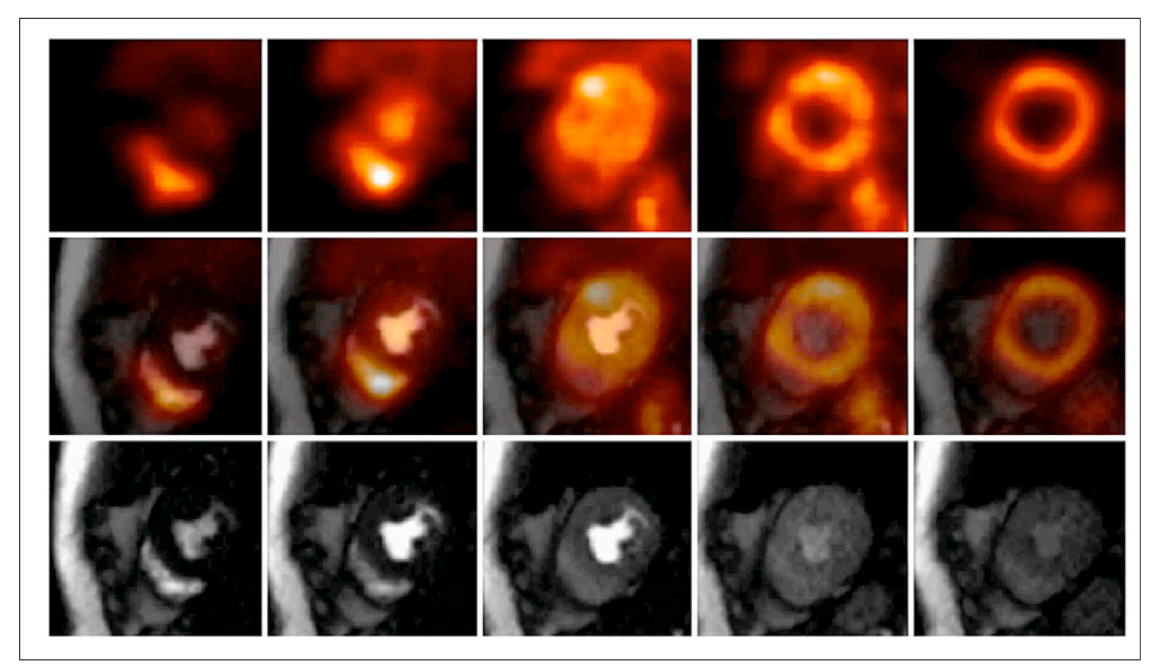


(T2* sequence), all of which are crucial for appropriate diagnosis. Although in ischemic cardiomyopathy fibrotic tissue is located in the subendocardium and follows the typical vascular distribution, in nonischemic cardiomyopathy no scar tissue, or primarily subepicardial fibrotic tissue, which does not follow the coronary artery distribution, is observed. Also, stress cardiac MR can be performed to assess for CAD.

Consequently, simultaneous assessment of the extent and exact localization of scar tissue by MR, as well as tissue perfusion and glucose metabolism by PET, improves the identification of the underlying disease.

Assessment of Myocardial Viability. The basic principle underlying myocardial viability imaging by PET is that hypoxia and ischemia lead to a shift in myocardial metabolism from oxidation of free fatty acids toward glucose utilization. Myocardium that is chronically hypoperfused or exposed to repetitive stunning, which brings about a state known as hibernation, shows chronic dysfunction and is known to shift toward glucose metabolism. The upregulated glucose utilization in ischemically compromised tissue has been linked to poor outcome (56), and the extent of the mismatched area has therapeutic significance (57-59). Viable but dysfunctional myocardium has the potential to recover after revascularization (60). Among the different imaging modalities available to assess myocardial viability, ${ }^{18} \mathrm{~F}-\mathrm{FDG}$ PET is considered to be the gold standard $(61,62)$. The results of several studies have proven the value of ${ }^{18}$ F-FDG PET imaging in identifying those patients who would benefit from revascularization, based on a prediction of improved LV function (63-65).

An important feature of PET lies in its potential to quantify tracer uptake, thus allowing differentiation between fully viable, partially viable, and nonviable myocardium, within the constraints imposed by spatial resolution. However, MR using the late gadolinium enhancement technique (LGE) is a valuable alternative to ${ }^{18} \mathrm{~F}$-FDG PET for myocardial viability imaging. The application of inversion-recovery prepared T1-weighted gradient-echo pulse sequences 5-20 min after administration of gadolinium-DTPA suppresses signal from remote myocardium and depicts scarred myocardium as enhanced areas in comparison to the surrounding normal and viable myocardium $(66,67)$. The inherently high in-plane resolution of MR allows the observer to distinguish between transmural and nontransmural enhancement, and even very small areas of subendocardial infarction can be detected. The presence of even a small amount of enhanced myocardium carries prognostic significance in patients with suspected CAD but without known prior myocardial infarction (68). A metaanalysis investigated the capabilities of imaging to predict functional recovery after revascularization; specificity for ${ }^{18}$ F-FDG PET and delayed-enhancement MR imaging was comparable $(63 \%)$, whereas the nuclear medicine technique had higher sensitivity (92\% vs. $84 \%)(61)$.

However, it is important to consider the fundamental differences between these imaging modalities; the increased extracellular space in scar tissue determines the
LGE signal, in contrast to the unambiguous relationship between ${ }^{18} \mathrm{~F}$-FDG uptake in PET, which represents a purely metabolic signal. Furthermore, PET allows the identification of compromised myocardium, thus adding therapeutically relevant information to the known relationship between infarct size and prognosis, whereas LGE reflects acute myocardial infarction or scarring at the site of prior infarction but not hibernating myocardium. From a study by Klein et al. it is known that the extent and location of delayed enhancement revealing the infarction scar correlates well with nonperfused, nonviable segments identified by PET (69). An example from our institution of covisualization of delayed-enhancement MR and viability assessed by ${ }^{18} \mathrm{~F}$-FDG PET from a patient after myocardial infarction is shown in Figure 4.

There may be segments classified as viable before revascularization for which metabolism and function fail to improve after revascularization. The integration of PET with structural and functional MR imaging markers such as diastolic wall thickness, contractile response on dobutamine stimulation, or the nontransmurality of LGE with a preserved rim of epicardial myocardium and preserved perfusion all might help to improve the viability-based prediction of dysfunctional segments after revascularization. This possibility suggests an interesting application for hybrid PET/MR scanners through simultaneous acquisition of myocardial perfusion and viability by PET while measuring by MR the LV function and LGE; this approach

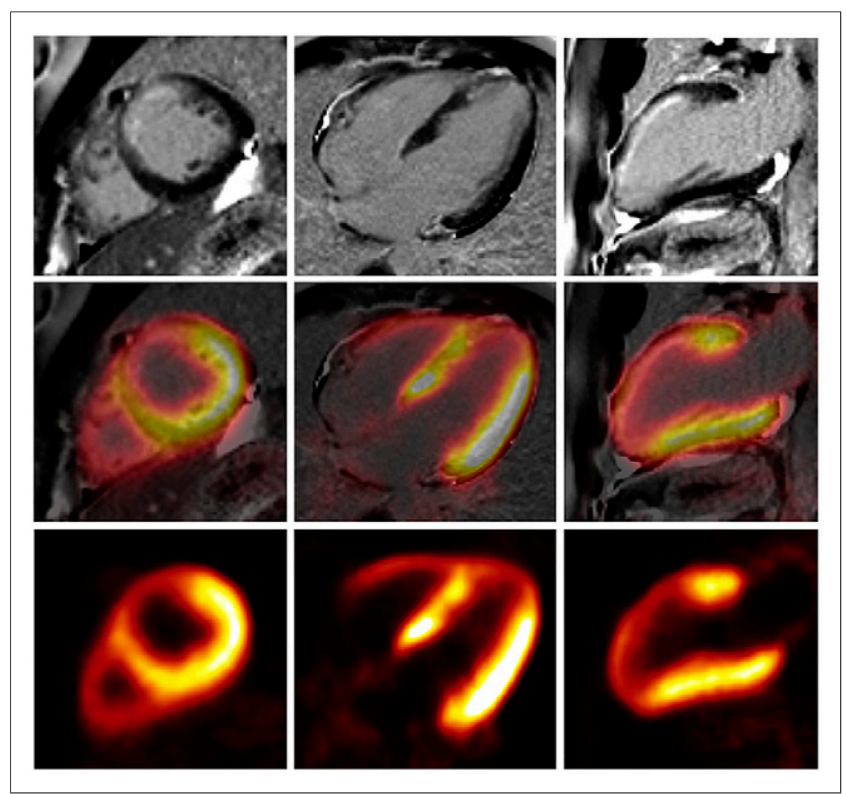

FIGURE 4. Images of delayed-enhancement MR (top), viability assessed by ${ }^{18} \mathrm{~F}$-FDG PET (bottom), and coregistration to structural image (middle) in patient with history of myocardial infarction. Images were obtained after euglycemic hyperinsulinemic clamping. In MR images, subendocardial enhancement of anterior wall and apex can be seen. These areas are well matched with reduced ${ }^{18} \mathrm{~F}-\mathrm{FDG}$ uptake in PET images. 
should yield new insights and may allow more reliable prediction of functional recovery after intervention $(70,71)$.

Inflammatory and Infiltrative Cardiomyopathies (Myocarditis and Sarcoidosis). Myocarditis is an inflammatory disease arising from various infections (primarily viral but also from bacteria, fungi, protozoa, or helminths), autoimmune processes, hypersensitivity reactions, and treatment with cardiotoxic drugs. Although invasive endomyocardial biopsy is the gold standard for the diagnosis of myocarditis, MR imaging is increasingly used for this purpose. The first case using this technique was published in 1991 by Gagliardi et al. (72). Since then, different protocols have been established, based on MR measures such as wall motion abnormalities, pericardial effusion, increased wall thickness, and changes in LV mass, which together represent the range of functional and morphologic abnormalities of the heart that can be depicted by MR imaging. However, MR imaging is also suited to image other pathologic abnormalities of the tissue in myocarditis, such as edema (T2-weighted imaging), hyperemia or capillary leakage (early gadolinium-enhanced T1-weighted imaging), and necrosis or fibrosis (late gadolinium-enhanced T1-weighted imaging). In a meta analysis including 194 patients, Friedrich et al. reported a diagnostic accuracy of $78 \%$ for MR-based imaging if at least 2 of the aforementioned tissue-based criteria were positive (73).

Even though the literature on myocarditis in PET imaging is scarce, a few potential applications should merit consideration. An increased number of apoptotic cells have been described in the heart of patients with myocarditis (74). Consequently, molecular imaging of apoptosis should be valuable for the diagnosis of myocarditis and potentially for risk stratification and prognosis. In a rat model of lipopolysaccharide-induced myocardial apoptosis, an observed increase in heart uptake of ${ }^{123} \mathrm{I}$-annexin $\mathrm{V}$ proved to be a caspase-dependent phenomenon (75). Annexin V represents a specific ligand for externalization of phosphatidylserine cells undergoing apoptotic changes (76). Caspases are also implicated in apoptosis, and caspase- 3 has recently been labeled with ${ }^{18} \mathrm{~F}$ (77). In a murine model of hepatic apoptosis, highly specific liver uptake of this tracer was shown (78). Further experiments determining the usefulness in cardiovascular applications of ${ }^{18} \mathrm{~F}$-labeled caspase-3 tracers seem justified.

By the hybrid PET/MR approach, imaging not only might confirm the diagnosis of myocarditis but might also-especially the PET part-be useful for assessing disease activity and thus guiding therapy.

Cardiac sarcoidosis represents another inflammatory cardiomyopathy for which integrated PET/MR imaging might be useful. In the case of cardiac involvement of sarcoidosis, the myocardium is replaced by fibrotic, fibrogranulomatous tissue. These fibrotic changes of the heart can be nicely imaged by MR using the LGE technique. In sarcoidosis, LGE shows a patchy distribution and appears primarily in subepicardial regions, being only seldom seen in the subendocardium. In PET imaging, different patterns of glucose metabolism (in the fasting state as a marker of inflammation) and myocardial perfusion are known. Intense glucose metabolism and normal perfusion indicate active inflammation, whereas reduced perfusion and high glucose metabolism represent an advanced stage of the disease. Absent or reduced perfusion and lack of glucose metabolism are indicative of end-stage cardiac sarcoidosis. An example of a patient with active cardiac involvement of sarcoidosis who underwent PET/MR is depicted in Figure 5.

Consequently, the integrated information from hybrid PET/MR not only allows assessment of the amount of affected myocardium by LGE but also helps with the assessment of the disease stage and might be valuable for therapy guidance.

\section{Advanced Molecular Imaging Applications}

Inflammatory Response After Acute Myocardial Infarction. An improved ability to predict LV remodeling after myocardial infarction might have important implications regarding therapy guidance. After the acute phase of myocardial infarction, the compromised tissue undergoes a healing process that involves inflammation, angiogenesis, fibroblast proliferation, and collagen deposition. Accumulating evidence indicates that an intense inflammatory response early after myocardial infarction may promote adverse remodeling and directly affect prognosis (79). This inflammatory reaction is driven mainly by different subsets of monocytes. Recently, Lee et al. proved that the noninvasive quantification of this inflammatory reaction by PET/MR is feasible in mice $(80)$. Our group also described a similar pattern of intense ${ }^{18} \mathrm{~F}-\mathrm{FDG}$ uptake in the infarct area despite the presence of transmural LGE in a rat model of transient ischemia (81). Furthermore, we observed the same pattern in patients who were scanned early after acute myocardial infarction in a fasted state (Fig. 6). Consequently, PET/MR has great potential to clarify the complex pathophysiologic healing process after MI and may help to predict LV remodeling in humans.

Atherosclerosis. An emerging application of MR in the cardiac field might be not in the primary diagnosis of CAD but in the closer characterization of atherosclerotic plaques.

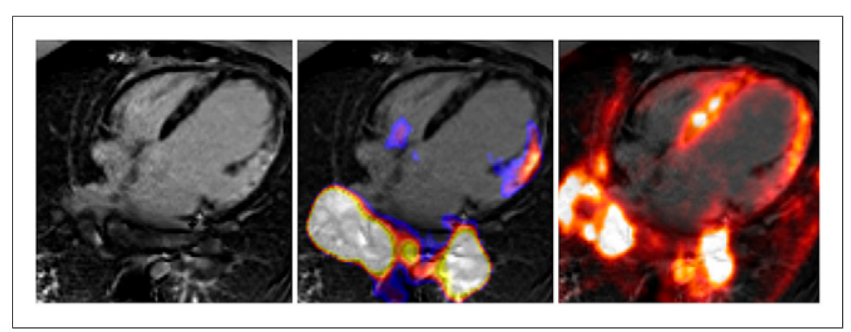

FIGURE 5. PET/MR images of patient who was referred to our institution because of suspected cardiac sarcoidosis. On 4-chamber view, patchy late gadolinium enhancement can be observed in basal lateral wall (left). In this region, upregulated glucose metabolism (middle) and reduced myocardial perfusion (right) can be observed indicating active inflammation. Also, increased ${ }^{18} \mathrm{~F}-\mathrm{FDG}$ uptake can be seen bilaterally in hilar lymph nodes. 


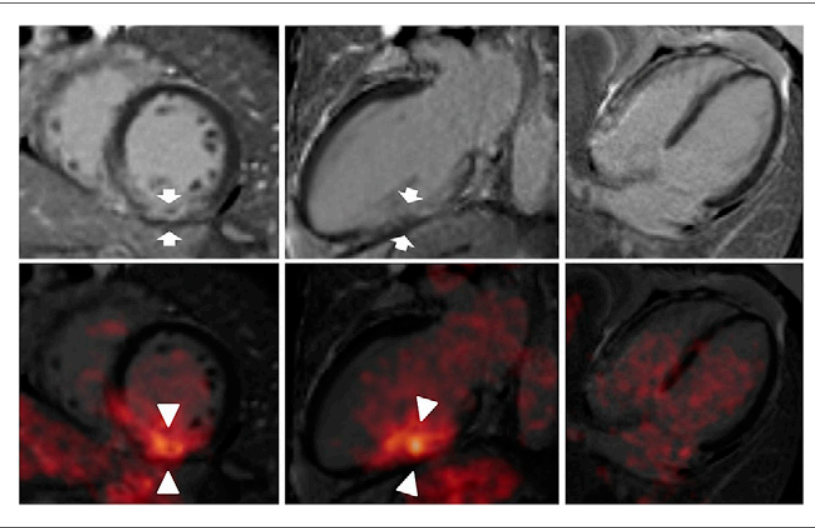

FIGURE 6. Images of delayed-enhancement MR (top) and coregistration of delayed-enhancement MR with ${ }^{18} \mathrm{~F}-\mathrm{FDG} \mathrm{PET}$ (bottom) from fasting patient who was imaged early after revascularization due to myocardial infarction. In MR images, subendocardial enhancement of basal inferior wall can be seen (arrows). ${ }^{18} \mathrm{~F}-\mathrm{FDG}$ PET images show increased uptake in corresponding area (arrowheads).

Fayad et al. showed for the first time that plaque imaging of the heart in humans is feasible (82). Direct visualization of the coronary arteries and thus assessment of wall thickness is becoming possible (83). Studies have proved that MR imaging is able to characterize several biochemically relevant parameters, such as water content, chemical composition, physical state, and molecular motion within the plaque (84).

These early findings for cardiac MR imaging of coronary plaques predict the capability of imaging longitudinally individual lesions and monitoring the effect of therapy, as well as bringing the capability of differentiating culprit from nonculprit lesions. In a study by von Bary et al., pigs with injury to the coronary wall by endothelial denudation or stent placement were imaged using a novel elastinbinding MR contrast agent, designated BMS-753951 (85). Delayed enhancement using BMS-753951 showed a 3-fold higher contrast-to-noise ratio in the stented coronary artery than in the balloon-injured or control (uninjured) artery, whereas gadolinium-DTPA showed only minor or no late enhancement. The authors concluded that this approach might be valuable for imaging the remodeling of human coronary arteries in patients with suspected CAD.

It is well established that inflammatory processes play a key role in the pathogenesis of atherosclerotic plaque formation and progression (86). ${ }^{18} \mathrm{~F}-\mathrm{FDG}$ PET shows increased tracer uptake in inflamed plaques and therefore has been proposed as a means to characterize the extent of inflammation in relation to macrophage infiltration $(87,88)$. Agents targeting integrin $\alpha_{V} \beta_{3}$ for the detection of plaque angiogenesis are additional promising PET tracers and are being investigated in preclinical models (89).

Characterization of the so-called vulnerable atherosclerotic plaque, which is at risk for disruption, is critical to predict and thus militate against acute adverse events. With
PET/MR, a multiparametric characterization of atherosclerotic plaques is now possible and may well have the potential to improve the identification of high-risk lesions in patients with atherosclerotic disease.

Another potential application of PET/MR imaging is 3-dimensional fusion of PET perfusion images with MRbased noninvasive angiography. This application would allow the exact localization of stenoses causing ischemia and would thus assist the cardiologist in the course of intervention. Furthermore, plaques not presently causing ischemia but fulfilling the criteria of a culprit lesion could be identified and selectively treated. An example of simultaneous ${ }^{18} \mathrm{~F}$-FDG PET/MR imaging in a patient with an atherosclerotic lesion in the carotid bifurcation is shown in Figure 7.

Stem Cell Therapy. Because of the death of cardiomyocytes after myocardial infarction, the affected myocardium undergoes loss of contractility and function, potentially leading to heart failure. The transplantation of stem cells is frequently mentioned as a promising therapeutic approach for cardiac regeneration. However, preclinical studies showed that only a fraction of the transplanted cells stay within the myocardium (90). PET/MR imaging might help us better understand the cellular and molecular mechanisms underlying cell engraftment. Also, improved cellular therapies might be validated with this new technique (91). A study by Higuchi et al. demonstrated that the combination of PET and MR imaging is suited for documenting both localization and survival of transplanted cells (92). In that study, human endothelial progenitor cells were labeled with iron oxide nanoparticles to impart MR contrast and were transformed using a viral vector to express the human sodium iodide symporter and then injected into the healthy

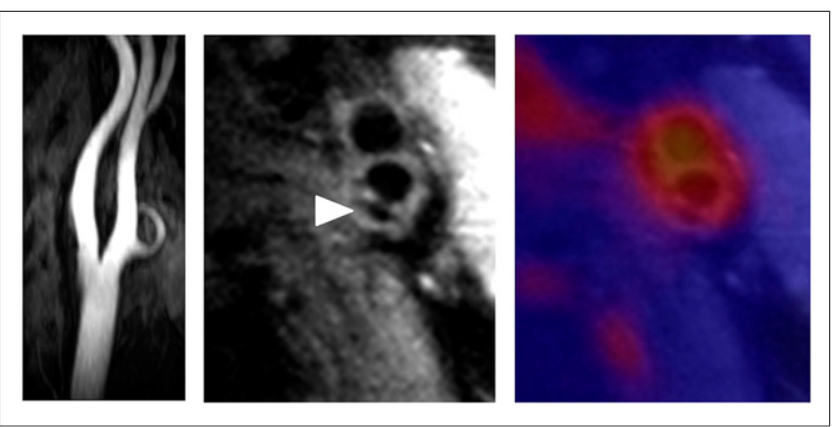

FIGURE 7. Simultaneous PET/MR imaging of atherosclerotic plaque in carotid artery. (Left) MR angiography shows no luminal stenosis. (Middle) High-resolution transverse MR image identifies atherosclerotic lesion at bifurcation with calcification (arrowhead). (Right) Fusion of PET (color) and MR images depicts enhanced ${ }^{18} \mathrm{~F}$ FDG tracer uptake within arterial vessel walls. Multimodal approach is able to comprehensively characterize this particular plaque: outward remodeling but no luminal stenosis is observed on MR imaging, which further characterizes plaque morphology by depicting overall plaque burden with area of calcification. Fused ${ }^{18} \mathrm{~F}-\mathrm{FDG}$ PET images reveal increased metabolic activity in carotid arteries, indicative of active inflammatory process. 
myocardium of nude rats, which lack a normal immune system. Although MR imaging proved to be well suited for the initial localization of the transplanted cells and for characterization of LV morphology, only PET imaging using ${ }^{124} \mathrm{I}$ as a substrate for human sodium iodide symporter was specific for imaging survival of the cells; the iron oxide particles were taken up by macrophages after death of the transplanted cells, such that the MR signal was not indicative of cell survival. The reporter gene imaging concept has been shown to be feasible also in large animals (93) but has scarcely been documented in humans.

These studies provide evidence that PET/MR hybrid imaging holds promise for use as a research tool in cell- or gene-based therapeutic approaches and may ultimately find applications in the clinic.

Neoangiogenesis. Neoangiogenesis seems to play a pivotal role after acute myocardial infarction. The prediction of LV remodeling might be assisted with ${ }^{18} \mathrm{~F}$-galactoarginine-glycine-aspartate (RGD), a PET tracer that binds to $\alpha_{\nu} \beta_{3}$ integrin receptors. Integrins are known to be of great importance for cell migration and for regulation of cell proliferation, survival, and differentiation. ${ }^{18} \mathrm{~F}$-galactoRGD has already been evaluated for the noninvasive assessment of $\alpha_{\nu} \beta_{3}$ integrin expression after myocardial infarction, both in an experimental setting using animals and in humans $(94,95)$. Recently, Sherif et al. published a report on the expression of $\alpha_{\nu} \beta_{3}$ integrin in the infarction area early after myocardial infarction in rats with ligation of the left anterior descending coronary artery (96). Interestingly, increased uptake of ${ }^{18} \mathrm{~F}$-galacto-RGD in the infarction area seemed to be a predictor for the absence of subsequent LV remodeling as assessed by MR imaging. However, further studies for the evaluation of this tracer need to be conducted.
PET/MR presents an ideal platform for detailed investigation of these processes given the capability to assess, among others, scar extent by LGE, LV function, and integrin expression by a single scan.

\section{CONCLUSION}

In this review, we have focused on applications in cardiac imaging in which both PET and MR imaging are routinely used in the clinic or have been validated in preclinical studies. Also, we have presented our initial experience with PET/MR in the field of cardiology, and our forecast provides our assessment of the future prospects for this imaging modality.

Table 4 summarizes the advantageous characteristics of combined PET/MR and its potential applications in cardiology. Both MR and PET have independently gained wide acceptance for the assessment of perfusion and tissue viability in patients with CAD. However, there are no obvious clinical indications or killer applications yet defined for the use of the integrated PET/MR instrumentation. Besides finding eventual clinical routine applications, PET/MR is presenting an attractive research platform that provides comprehensive phenotyping of cardiovascular diseases. Parallel imaging using integrated PET/MR instrumentation allows for the elegant cross-validation of new imaging methods under one and the same physiologic condition. It seems clear that PET/MR will increase workflow complexity and that it can reduce radiation exposure by obviating CT-based attenuation correction, although the technical difficulties arising are formidable. Ultimately, the great strength of PET/MR will lie in its ability to deliver multimodal quantitative imaging parameters based on dynamic data acquisition in both channels. Continuous motion correction provided

TABLE 4

Advantageous Features of Combined PET/MR with Focus on Cardiac Applications

\begin{tabular}{|c|c|}
\hline Assessment of. .. & Advantageous features of PET/MR \\
\hline Morphology & $\begin{array}{l}\text { No ionizing radiation, high soft-tissue contrast, coronaries }+ \text {, plaque } \\
\text { imaging }+ \text {, cardiac structure }+++ \text {, fiber architecture }+++\end{array}$ \\
\hline LV function & $\begin{array}{l}\text { Gold standard; correlation with metabolic information/perfusion (risk } \\
\text { stratification, prognosis) }\end{array}$ \\
\hline Perfusion & $\begin{array}{l}\text { Cross-validation of myocardial blood flow quantification; no contrast } \\
\text { agents for arterial spin-labeled MR imaging; attenuation correction } \\
\text { by MR; motion and partial-volume correction for myocardial blood } \\
\text { flow quantification }\end{array}$ \\
\hline Infarction and viability & $\begin{array}{l}\text { Scar delineation (LGE) }+++ \text {; combination of glucose metabolism } \\
\text { (viable vs. nonviable), perfusion (normal perfusion, hypoperfusion, no } \\
\text { perfusion), LV parameters, and LGE (transmural vs. nontransmural } \\
\text { scar); potential additional value for risk stratification/prognosis/ } \\
\text { therapy guidance }\end{array}$ \\
\hline $\begin{array}{l}\text { Molecular imaging (e.g., inflammation, } \\
\text { angiogenesis, sympathetic innervation, } \\
\text { gene transfer, and cell transplantation) }\end{array}$ & $\begin{array}{l}\text { MR spectroscopy (cardiac metabolism and composition); ideal } \\
\text { combination of high sensitivity of PET and vast variety of PET } \\
\text { radiotracers for detection and quantification of molecular targets as } \\
\text { well as localization and volume correction by MR; still large scope } \\
\text { for development of new tracers, imaging techniques, and } \\
\text { applications in MR }\end{array}$ \\
\hline
\end{tabular}


by MR imaging may improve substantially the quantification of regional PET tracer uptake. Access to coregistered, almost simultaneous physiologic and biologic measurements have already made PET/MR the most sophisticated quantitative imaging modality in cardiology, although these applications are in their infancy.

The financial aspects of MR versus $\mathrm{CT}$ as an adjunct to PET need to be considered. PET/MR is entering routine clinical practice at present, but it is still not clear whether PET/MR will be able to offer added value or generate an additional demand for imaging studies that PET/CT cannot satisfy. Thus, whether the higher acquisition costs for PET/ MR will pay off in the long-term still needs to be proven. However, we expect that the combination of molecular imaging with superb functional characterization of cardiac performance will help to stage heart failure and to develop predictive parameters for tissue recovery and response to therapy. The results of such sophisticated research applications will define new clinical indications, which need to be validated as superior and cost-effective replacements for existing imaging approaches in cardiology.

\section{ACKNOWLEDGMENTS}

This review has been prepared with the help of many staff members, but we would like to thank especially Shelley Zhang and Sebastian Fürst for valuable input, Sylvia Schachoff and Brigitte Dzewas for their technical assistance during PET/MR acquisitions, and Dr. Paul Cumming for professional textual revisions.

\section{REFERENCES}

1. Schwaiger M, Ziegler S, Nekolla SG. PET/CT: challenge for nuclear cardiology. J Nucl Med. 2005;46:1664-1678.

2. Kajander S, Ukkonen H, Sipila H, Teras M, Knuuti J. Low radiation dose imaging of myocardial perfusion and coronary angiography with a hybrid PET/CT scanner. Clin Physiol Funct Imaging. 2009;29:81-88.

3. Koepfli P, Hany TF, Wyss CA, et al. CT attenuation correction for myocardial perfusion quantification using a PET/CT hybrid scanner. J Nucl Med. 2004;45:537-542.

4. Fink C, Krissak R, Henzler T, et al. Radiation dose at coronary CT angiography: second-generation dual-source CT versus single-source 64-MDCT and first-generation dual-source CT. AJR. 2011;196:W550-W557.

5. Bellenger NG, Davies LC, Francis JM, Coats AJ, Pennell DJ. Reduction in sample size for studies of remodeling in heart failure by the use of cardiovascular magnetic resonance. J Cardiovasc Magn Reson. 2000;2:271-278.

6. Greupner J, Zimmermann E, Grohmann A, et al. Head-to-head comparison of left ventricular function assessment with 64-row computed tomography, biplane left cineventriculography, and both 2- and 3-dimensional transthoracic echocardiography: comparison with magnetic resonance imaging as the reference standard. J Am Coll Cardiol. 2012;59:1897-1907.

7. Zhang H, Shea SM, Park V, et al. Accurate myocardial T1 measurements: toward quantification of myocardial blood flow with arterial spin labeling. Magn Reson Med. 2005;53:1135-1142.

8. Zun Z, Varadarajan P, Pai RG, Wong EC, Nayak KS. Arterial spin labeled CMR detects clinically relevant increase in myocardial blood flow with vasodilation. JACC Cardiovasc Imaging. 2011;4:1253-1261.

9. Spiro AJ, Haramati LB, Jain VR, Godelman A, Travin MI, Levsky JM. Resting cardiac 64-MDCT does not reliably detect myocardial ischemia identified by radionuclide imaging. AJR. 2013;200:337-342.

10. Bottomley PA, Weiss RG. Non-invasive magnetic-resonance detection of creatine depletion in non-viable infarcted myocardium. Lancet. 1998;351:714-718.

11. Szczepaniak LS, Dobbins RL, Metzger GJ, et al. Myocardial triglycerides and systolic function in humans: in vivo evaluation by localized proton spectroscopy and cardiac imaging. Magn Reson Med. 2003;49:417-423.
12. Jansen MA, Van Emous JG, Nederhoff MG, Van Echteld CJ. Assessment of myocardial viability by intracellular ${ }^{23} \mathrm{Na}$ magnetic resonance imaging. Circulation. 2004;110:3457-3464.

13. Wedeen VJ, Hagmann P, Tseng WY, Reese TG, Weisskoff RM. Mapping complex tissue architecture with diffusion spectrum magnetic resonance imaging. Magn Reson Med. 2005;54:1377-1386.

14. Sosnovik DE, Wang R, Dai G, et al. Diffusion spectrum MR imaging tractography reveals the presence of a complex network of residual myofibers in infarcted myocardium. Circ Cardiovasc Imaging. 2009;2:206-212.

15. Antoch G, Bockisch A. Combined PET/MR imaging: a new dimension in wholebody oncology imaging? Eur J Nucl Med Mol Imaging. 2009;36(suppl 1):S113S120.

16. Nekolla SG, Martinez-Moeller A, Saraste A. PET and MR imaging in cardiac imaging: from validation studies to integrated applications. Eur J Nucl Med Mol Imaging. 2009;36(suppl 1):S121-S130.

17. Zaidi H, Ojha N, Morich M, et al. Design and performance evaluation of a whole-body Ingenuity TF PET-MR imaging system. Phys Med Biol. 2011;56:3091-3106.

18. Delso G, Furst S, Jakoby B, et al. Performance measurements of the Siemens mMR integrated whole-body PET/MR scanner. J Nucl Med. 2011;52:19141922.

19. Hofmann M, Pichler B, Scholkopf B, Beyer T. Towards quantitative PET/MR imaging: a review of MR-based attenuation correction techniques. Eur J Nucl Med Mol Imaging. 2009;36(suppl 1):S93-S104.

20. Martinez-Möller A, Souvatzoglou M, Navab N, Schwaiger M, Nekolla SG. Artifacts from misaligned CT in cardiac perfusion PET/CT studies: frequency, effects, and potential solutions. J Nucl Med. 2007;48:188-193.

21. Huang SC, Carson RE, Phelps ME, Hoffman EJ, Schelbert HR, Kuhl DE. A boundary method for attenuation correction in positron computed tomography. $J$ Nucl Med. 1981;22:627-637.

22. Martinez-Möller A, Souvatzoglou M, Delso G, et al. Tissue classification as a potential approach for attenuation correction in whole-body PET/MR imaging: evaluation with PET/CT data. J Nucl Med. 2009;50:520-526.

23. Coombs BD, Szumowski J, Coshow W. Two-point Dixon technique for water-fat signal decomposition with B0 inhomogeneity correction. Magn Reson Med. 1997;38:884-889.

24. Schulz V, Torres-Espallardo I, Renisch S, et al. Automatic, three-segment, MRbased attenuation correction for whole-body PET/MR data. Eur J Nucl Med Mol Imaging. 2011;38:138-152.

25. Samarin A, Burger C, Wollenweber SD, et al. PET/MR imaging of bone lesions: implications for PET quantification from imperfect attenuation correction. Eur J Nucl Med Mol Imaging. 2012;39:1154-1160.

26. Fürst S, Souvatzoglu M, Rischpler C, Ziegler S, Schwaiger M, Nekolla S. Effects of MR contrast agents on attenuation map generation and cardiac PET quantification in PET/MR [abstract]. J Nucl Med. 2012;53(suppl 1):43P.

27. Drzezga A, Souvatzoglou M, Eiber M, et al. First clinical experience with integrated whole-body PET/MR: comparison to PET/CT in patients with oncologic diagnoses. J Nucl Med. 2012;53:845-855.

28. Hofmann M, Bezrukov I, Mantlik F, et al. MR imaging-based attenuation correction for whole-body PET/MR imaging: quantitative evaluation of segmentation- and atlas-based methods. J Nucl Med. 2011;52:1392-1399.

29. Nuyts J, Dupont P, Stroobants S, Bennick R, Mortelmans L, Suetens P. Simultaneous maximum a posteriori reconstruction of attenuation and activity distributions from emission sinograms. IEEE Trans Med Imaging. 1999;18(5):393-403.

30. Nuyts J, Bal G, Kehren F, Fenchel M, Michel C, Watson C. Completion of a truncated attenuation image from the attenuated PET emission data. IEEE Trans Med Imaging. September 21, 2012 [Epub ahead of print].

31. Delso G, Martinez-Moller A, Bundschuh RA, Nekolla SG, Ziegler SI. The effect of limited MR field of view in MR/PET attenuation correction. Med Phys. 2010;37:2804-2812.

32. Blumhagen JO, Ladebeck R, Fenchel M, Scheffler K. MR-based field-of-view extension in MR/PET: $\mathrm{B}(0)$ homogenization using gradient enhancement (HUGE). Magn Reson Med. November 30, 2012 [Epub ahead of print].

33. Delso G, Martinez-Moller A, Bundschuh RA, et al. Evaluation of the attenuation properties of MR equipment for its use in a whole-body PET/MR scanner. Phys Med Biol. 2010;55:4361-4374.

34. MacDonald LR, Kohlmyer S, Liu C, Lewellen TK, Kinahan PE. Effects of MR surface coils on PET quantification. Med Phys. 2011;38:2948-2956.

35. Nekolla S, Souvatzoglou M, Schachoff S, et al. MR/PET attenuation correction using a multi tissue model for quantification of cardiac uptake: initial comparison to PET/CT [abstract]. J Nucl Med. 2011;52(suppl 1):130P.

36. Adluru G, Chen L, Kim SE, et al. Three-dimensional late gadolinium enhancement imaging of the left atrium with a hybrid radial acquisition and compressed sensing. J Magn Reson Imaging. 2011;34:1465-1471. 
37. Xue H, Zuehlsdorff S, Kellman P, et al. Unsupervised inline analysis of cardiac perfusion MR imaging. Med Image Comput Comput Assist Interv. 2009;12:741749.

38. Klocke FJ, Baird MG, Lorell BH, et al. ACC/AHA/ASNC guidelines for the clinical use of cardiac radionuclide imaging: executive summary-a report of the American College of Cardiology/American Heart Association Task Force on Practice Guidelines (ACC/AHA/ASNC Committee to Revise the 1995 Guidelines for the Clinical Use of Cardiac Radionuclide Imaging). J Am Coll Cardiol. 2003;42:1318-1333.

39. Parker MW, Iskandar A, Limone B, et al. Diagnostic accuracy of cardiac positron emission tomography versus single photon emission computed tomography for coronary artery disease: a bivariate meta-analysis. Circ Cardiovasc Imaging. 2012;5:700-707.

40. McArdle BA, Dowsley TF, deKemp RA, Wells GA, Beanlands RS. Does rubidium82 PET have superior accuracy to SPECT perfusion imaging for the diagnosis of obstructive coronary disease? A systematic review and meta-analysis. J Am Coll Cardiol. 2012;60:1828-1837.

41. Fukushima K, Javadi MS, Higuchi T, et al. Prediction of short-term cardiovascular events using quantification of global myocardial flow reserve in patients referred for clinical ${ }^{82} \mathrm{Rb}$ PET perfusion imaging. J Nucl Med. 2011;52:726-732.

42. Yoshinaga K, Chow BJ, Williams K, et al. What is the prognostic value of myocardial perfusion imaging using rubidium-82 positron emission tomography? J Am Coll Cardiol. 2006;48:1029-1039.

43. Merhige ME, Breen WJ, Shelton V, Houston T, D'Arcy BJ, Perna AF. Impact of myocardial perfusion imaging with $\mathrm{PET}$ and ${ }^{82} \mathrm{Rb}$ on downstream invasive procedure utilization, costs, and outcomes in coronary disease management. $\mathrm{J} \mathrm{Nucl}$ Med. 2007;48:1069-1076.

44. Hachamovitch R, Hayes SW, Friedman JD, Cohen I, Berman DS. Comparison of the short-term survival benefit associated with revascularization compared with medical therapy in patients with no prior coronary artery disease undergoing stress myocardial perfusion single photon emission computed tomography. Circulation. 2003;107:2900-2907.

45. Manning WJ, Atkinson DJ, Grossman W, Paulin S, Edelman RR. First-pass nuclear magnetic resonance imaging studies using gadolinium-DTPA in patients with coronary artery disease. J Am Coll Cardiol. 1991;18:959-965.

46. Nandalur KR, Dwamena BA, Choudhri AF, Nandalur MR, Carlos RC. Diagnostic performance of stress cardiac magnetic resonance imaging in the detection of coronary artery disease: a meta-analysis. J Am Coll Cardiol. 2007;50:13431353.

47. de Jong MC, Genders TS, van Geuns RJ, Moelker A, Hunink MG. Diagnostic performance of stress myocardial perfusion imaging for coronary artery disease: a systematic review and meta-analysis. Eur Radiol. 2012;22:1881-1895.

48. Parkash R, deKemp RA, Ruddy TD, et al. Potential utility of rubidium 82 PET quantification in patients with 3 -vessel coronary artery disease. $J$ Nucl Cardiol. 2004;11:440-449.

49. Kajander SA, Joutsiniemi E, Saraste M, et al. Clinical value of absolute quantification of myocardial perfusion with ${ }^{15} \mathrm{O}$-water in coronary artery disease. Circ Cardiovasc Imaging. 2011;4:678-684.

50. Schwaiger M, Melin J. Cardiological applications of nuclear medicine. Lancet. 1999;354:661-666.

51. Rischpler C, Park MJ, Fung GS, Javadi M, Tsui BM, Higuchi T. Advances in PET myocardial perfusion imaging: F-18 labeled tracers. Ann Nucl Med. 2012;26:1-6.

52. Schwitter J, Nanz D, Kneifel S, et al. Assessment of myocardial perfusion in coronary artery disease by magnetic resonance: a comparison with positron emission tomography and coronary angiography. Circulation. 2001;103:22302235.

53. Ibrahim T, Nekolla SG, Schreiber K, et al. Assessment of coronary flow reserve: comparison between contrast-enhanced magnetic resonance imaging and positron emission tomography. J Am Coll Cardiol. 2002;39:864-870.

54. O'Neill JO, McCarthy PM, Brunken RC, et al. PET abnormalities in patients with nonischemic cardiomyopathy. J Card Fail. 2004;10:244-249.

55. Eisenberg JD, Sobel BE, Geltman EM. Differentiation of ischemic from nonischemic cardiomyopathy with positron emission tomography. Am J Cardiol. 1987;59:1410-1414

56. Beanlands RS, Hendry PJ, Masters RG, deKemp RA, Woodend K, Ruddy TD. Delay in revascularization is associated with increased mortality rate in patients with severe left ventricular dysfunction and viable myocardium on fluorine 18fluorodeoxyglucose positron emission tomography imaging. Circulation. 1998; 98:II51-II56.

57. Di Carli MF, Davidson M, Little R, et al. Value of metabolic imaging with positron emission tomography for evaluating prognosis in patients with coronary artery disease and left ventricular dysfunction. Am J Cardiol. 1994;73:527-533.
58. D'Egidio G, Nichol G, Williams KA, et al. Increasing benefit from revascularization is associated with increasing amounts of myocardial hibernation: a substudy of the PARR-2 trial. JACC Cardiovasc Imaging. 2009;2:1060-1068.

59. Allman KC, Shaw LJ, Hachamovitch R, Udelson JE. Myocardial viability testing and impact of revascularization on prognosis in patients with coronary artery disease and left ventricular dysfunction: a meta-analysis. J Am Coll Cardiol. 2002;39:1151-1158.

60. Heyndrickx GR, Millard RW, McRitchie RJ, Maroko PR, Vatner SF. Regional myocardial functional and electrophysiological alterations after brief coronary artery occlusion in conscious dogs. J Clin Invest. 1975;56:978-985.

61. Schinkel AF, Poldermans D, Elhendy A, Bax JJ. Assessment of myocardial viability in patients with heart failure. J Nucl Med. 2007;48:1135-1146.

62. Ghosh N, Rimoldi OE, Beanlands RS, Camici PG. Assessment of myocardial ischaemia and viability: role of positron emission tomography. Eur Heart J. 2010;31:2984-2995.

63. Schinkel AF, Bax JJ, Poldermans D, Elhendy A, Ferrari R, Rahimtoola SH. Hibernating myocardium: diagnosis and patient outcomes. Curr Probl Cardiol. 2007;32:375-410.

64. vom Dahl J, Eitzman DT, al-Aouar ZR, et al. Relation of regional function, perfusion, and metabolism in patients with advanced coronary artery disease undergoing surgical revascularization. Circulation. 1994;90:2356-2366.

65. Tillisch J, Brunken R, Marshall R, et al. Reversibility of cardiac wall-motion abnormalities predicted by positron tomography. N Engl J Med. 1986;314:884888.

66. Klein C, Nekolla SG, Balbach T, et al. The influence of myocardial blood flow and volume of distribution on late Gd-DTPA kinetics in ischemic heart failure. $J$ Magn Reson Imaging. 2004;20:588-593.

67. Klein C, Schmal TR, Nekolla SG, Schnackenburg B, Fleck E, Nagel E. Mechanism of late gadolinium enhancement in patients with acute myocardial infarction. J Cardiovasc Magn Reson. 2007;9:653-658.

68. Kwong RY, Chan AK, Brown KA, et al. Impact of unrecognized myocardial scar detected by cardiac magnetic resonance imaging on event-free survival in patients presenting with signs or symptoms of coronary artery disease. Circulation. 2006;113:2733-2743.

69. Klein C, Nekolla SG, Bengel FM, et al. Assessment of myocardial viability with contrast-enhanced magnetic resonance imaging: comparison with positron emission tomography. Circulation. 2002;105:162-167.

70. Schmidt M, Voth E, Schneider CA, et al. F-18-FDG uptake is a reliable predictory of functional recovery of akinetic but viable infarct regions as defined by magnetic resonance imaging before and after revascularization. Magn Reson Imaging. 2004;22:229-236.

71. Gerber BL, Rochitte CE, Bluemke DA, et al. Relation between Gd-DTPA contrast enhancement and regional inotropic response in the periphery and center of myocardial infarction. Circulation. 2001;104:998-1004.

72. Gagliardi MG, Bevilacqua M, Di Renzi P, Picardo S, Passariello R, Marcelletti C. Usefulness of magnetic resonance imaging for diagnosis of acute myocarditis in infants and children, and comparison with endomyocardial biopsy. Am J Cardiol. 1991;68:1089-1091.

73. Friedrich MG, Sechtem U, Schulz-Menger J, et al. Cardiovascular magnetic resonance in myocarditis: a JACC white paper. J Am Coll Cardiol. 2009; 53:1475-1487.

74. Alter P, Jobmann M, Meyer E, Pankuweit S, Maisch B. Apoptosis in myocarditis and dilated cardiomyopathy: does enterovirus genome persistence protect from apoptosis? An endomyocardial biopsy study. Cardiovasc Pathol. 2001;10:229234.

75. Pétillot $\mathrm{P}$, Lahorte C, Bonanno E, et al. Annexin V detection of lipopolysaccharide-induced cardiac apoptosis. Shock. 2007;27:69-74.

76. Gerke V, Moss SE. Annexins: from structure to function. Physiol Rev. 2002; 82:331-371.

77. Faust A, Wagner S, Law MP, et al. The nonpeptidyl caspase binding radioligand (S)-1-(4-(2-[ ${ }^{18}$ F]Fluoroethoxy)-benzyl)-5-[1-(2-methoxymethylpyrrolidinyl)sulfonyl $]$ isatin $\left(\left[{ }^{18} \mathrm{~F}\right] \mathrm{CbR}\right)$ as potential positron emission tomography-compatible apoptosis imaging agent. Q J Nucl Med Mol Imaging. 2007;51:67-73.

78. Zhou D, Chu W, Rothfuss J, et al. Synthesis, radiolabeling, and in vivo evaluation of an ${ }^{18} \mathrm{~F}$-labeled isatin analog for imaging caspase-3 activation in apoptosis. Bioorg Med Chem Lett. 2006;16:5041-5046.

79. van der Laan AM, Nahrendorf M, Piek JJ. Healing and adverse remodelling after acute myocardial infarction: role of the cellular immune response. Heart. 2012;98:1384-1390.

80. Lee WW, Marinelli B, van der Laan AM, et al. PET/MR imaging of inflammation in myocardial infarction. J Am Coll Cardiol. 2012;59:153-163.

81. Higuchi T, Nekolla SG, Jankaukas A, et al. Characterization of normal and infarcted rat myocardium using a combination of small-animal PET and clinical MR imaging. J Nucl Med. 2007;48:288-294. 
82. Fayad ZA, Fuster V, Fallon JT, et al. Noninvasive in vivo human coronary artery lumen and wall imaging using black-blood magnetic resonance imaging. Circulation. 2000;102:506-510.

83. Kim WY, Stuber M, Bornert P, Kissinger KV, Manning WJ, Botnar RM. Threedimensional black-blood cardiac magnetic resonance coronary vessel wall imaging detects positive arterial remodeling in patients with nonsignificant coronary artery disease. Circulation. 2002;106:296-299.

84. Fuster V, Kim RJ. Frontiers in cardiovascular magnetic resonance. Circulation. 2005;112:135-144.

85. von Bary C, Makowski M, Preissel A, et al. MR imaging of coronary wall remodeling in a swine model of coronary injury using an elastin-binding contrast agent. Circ Cardiovasc Imaging. 2011;4:147-155.

86. Ross R. Atherosclerosis: an inflammatory disease. N Engl J Med. 1999;340:115126.

87. Rudd JH, Warburton EA, Fryer TD, et al. Imaging atherosclerotic plaque inflammation with $\left[{ }^{18} \mathrm{~F}\right]$-fluorodeoxyglucose positron emission tomography. Circulation. 2002;105:2708-2711.

88. Davies JR, Rudd JH, Weissberg PL, Narula J. Radionuclide imaging for the detection of inflammation in vulnerable plaques. J Am Coll Cardiol. 2006;47: C57-C68.

89. Laitinen I, Saraste A, Weidl E, et al. Evaluation of alphavbeta3 integrin-targeted positron emission tomography tracer ${ }^{18} \mathrm{~F}$-galacto-RGD for imaging of vascular inflammation in atherosclerotic mice. Circ Cardiovasc Imaging. 2009;2:331338.

90. Terrovitis J, Lautamaki R, Bonios M, et al. Noninvasive quantification and optimization of acute cell retention by in vivo positron emission tomography after intramyocardial cardiac-derived stem cell delivery. J Am Coll Cardiol. 2009;54:1619-1626.

91. Zhang SJ, Wu JC. Comparison of imaging techniques for tracking cardiac stem cell therapy. J Nucl Med. 2007;48:1916-1919.

92. Higuchi T, Anton M, Dumler K, et al. Combined reporter gene PET and iron oxide MR imaging for monitoring survival and localization of transplanted cells in the rat heart. $J$ Nucl Med. 2009;50:1088-1094.

93. Bengel FM, Anton M, Richter T, et al. Noninvasive imaging of transgene expression by use of positron emission tomography in a pig model of myocardial gene transfer. Circulation. 2003;108:2127-2133.

94. Higuchi T, Bengel FM, Seidl S, et al. Assessment of alphavbeta3 integrin expression after myocardial infarction by positron emission tomography. Cardiovasc Res. 2008;78:395-403.

95. Makowski MR, Ebersberger U, Nekolla S, Schwaiger M. In vivo molecular imaging of angiogenesis, targeting alphavbeta 3 integrin expression, in a patient after acute myocardial infarction. Eur Heart J. 2008;29:2201.

96. Sherif HM, Saraste A, Nekolla SG, et al. Molecular imaging of early alphavbeta 3 integrin expression predicts long-term left-ventricle remodeling after myocardial infarction in rats. $J$ Nucl Med. 2012;53:318-323. 